\title{
Type-Il quantum-dot-in-nanowire structures with large oscillator strength for optical quantum gate applications
}

Taherkhani, Masoomeh; Willatzen, Morten; Mørk, Jesper; Gregersen, Niels; McCutcheon, Dara P. S.

Published in:

Physical Review B

Link to article, DOI:

10.1103/PhysRevB.96.125408

Publication date:

2017

Document Version

Publisher's PDF, also known as Version of record

Link back to DTU Orbit

Citation (APA):

Taherkhani, M., Willatzen, M., Mørk, J., Gregersen, N., \& McCutcheon, D. P. S. (2017). Type-II quantum-dot-innanowire structures with large oscillator strength for optical quantum gate applications. Physical Review $B$, 96(12), [125408]. https://doi.org/10.1103/PhysRevB.96.125408

\section{General rights}

Copyright and moral rights for the publications made accessible in the public portal are retained by the authors and/or other copyright owners and it is a condition of accessing publications that users recognise and abide by the legal requirements associated with these rights.

- Users may download and print one copy of any publication from the public portal for the purpose of private study or research.

- You may not further distribute the material or use it for any profit-making activity or commercial gain

- You may freely distribute the URL identifying the publication in the public portal 


\title{
Type-II quantum-dot-in-nanowire structures with large oscillator strength for optical quantum gate applications
}

\author{
Masoomeh Taherkhani, ${ }^{*}$ Morten Willatzen, Jesper Mørk, and Niels Gregersen \\ Department of Photonics Engineering, Technical University of Denmark, Ørsteds Plads Building 345A, \\ 2800 Kongens Lyngby, Denmark \\ Dara P. S. McCutcheon \\ Quantum Engineering Technology Labs, H. H. Wills Physics Laboratory and Department of Electrical \\ and Electronic Engineering, University of Bristol, BS8 1FD, United Kingdom \\ (Received 28 July 2017; revised manuscript received 22 August 2017; published 7 September 2017)
}

\begin{abstract}
We present a numerical investigation of the exciton energy and oscillator strength in type-II nanowire quantum dots. For a single quantum dot, the poor overlap of the electron part and the weakly confined hole part of the excitonic wave function leads to a low oscillator strength compared to type-I systems. To increase the oscillator strength, we propose a double quantum dot structure featuring a strongly localized exciton wave function and a corresponding fourfold relative enhancement of the oscillator strength, paving the way towards efficient optically controlled quantum gate applications in the type-II nanowire system. The simulations are performed using a computationally efficient configuration-interaction method suitable for handling the relatively large nanowire structures.
\end{abstract}

DOI: 10.1103/PhysRevB.96.125408

\section{INTRODUCTION}

The quantum dot (QD) with its discrete spectrum of energy levels, similar to those of atoms, represents a highly promising candidate for implementing all-optical quantum gates for quantum information processing [1-3]. Using ultrafast laser pulses, excitons in QDs can be individually addressed and coherently manipulated on a time scale shorter than the typical dephasing time [4]. The excitons' strong quantum confinement in QDs suppresses coupling to the solid-state environment $[5,6]$ and more importantly, it greatly enhances the Coulomb interaction/excitonic effect which provides a mean to perform two-qubit operations $[7,8]$.

In the following, we will consider QDs implemented in a rotationally symmetric nanowire as illustrated in Fig. 1(a). We distinguish between two types of QDs. In type-I QDs, the band gap of one material is contained entirely within that of another. In this case, both the electron and the hole are confined in the material with the narrower band gap as shown in Fig. 1(b) for the double quantum dot (DQD) configuration. However, in the recently emerging type-II QD systems [9], both the conduction and the valence band edges of one material lie above the corresponding edges of the neighboring material, which typically results in the electron and hole being confined in different regions as shown in Fig. 1(c). In this configuration, the electron is confined inside the QDs, with equal probability to be found in each of them, while the hole is confined between QDs. This spatial distribution of the electron and the hole on different sites leads to a reduced electron-hole overlap and a smaller oscillator strength of the exciton in these structures compared to that of type-I QDs [10].

When encoding the qubits on the excitons of neighboring QDs, two-qubit operations can be performed using controlled interaction of the excitons in the QDs. In the type-I DQD configuration, the interaction between two excitons confined to different QDs as shown in Fig. 1(b) is weak even when

*Corresponding author: masoomehtaherkhani@gmail.com two QDs are in close proximity to each other, and an external electric field is needed in these systems to make the excitons interact with each other [11]. In contrast, in a type-II DQD structure, since the hole state is confined between two QDs as illustrated in Fig. 1(c), interaction is possible even in the absence of an external electric field or when the QDs are far from each other. This feature makes type-II QDs a promising structure for implementing quantum gates.

Extensive studies of exciton properties in type-I QDs have been conducted [12-14], and exploitation of type-I QD structures for quantum gate operations have been proposed $[7,15,16]$. However, studies of the optical properties of type-II QDs have only recently been initiated [17-20], and a detailed scheme for using type-II QDs for implementing quantum gates has not yet been proposed to the best of our knowledge.

In this paper we report the optical properties of type-II crystal-phase QDs in an InP nanowire. Figure 2(a) shows a scanning electron microscope (SEM) image of InP nanowire samples, while Fig. 2(b) illustrates crystal-phase QDs in a single nanowire. The QDs are formed by a transition in the crystallographic lattice of the InP material between the zinc blende and wurtzite crystal phases. The transitions between the two phases are induced using temperature control during the growth process [21]. This structuring, with the same material composition of InP everywhere, can be made with atomic monolayer precision, and this good control of the geometry makes crystal-phase QDs suitable systems for engineering QD-based functionalities such as quantum gates [22,23].

The accurate modeling of excitons in a QD-nanowire system taking into account detailed electronic band structures is possible using methods such as multiband $k \cdot p$ theory, $a b$ initio, and tight-binding calculations [21,24,25]. However, in this work we perform geometrical parameter sweeps to analyze and optimize the device performance, and for this reason we prefer to use a less demanding single-band model based on the envelope function and effective mass approximations. We include Coulomb interaction in our model by employing a full configuration-interaction description of the 
(a)
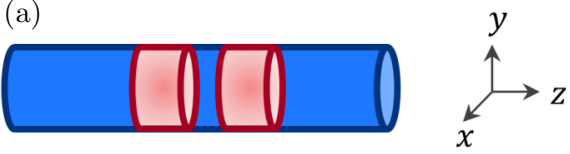

(b)

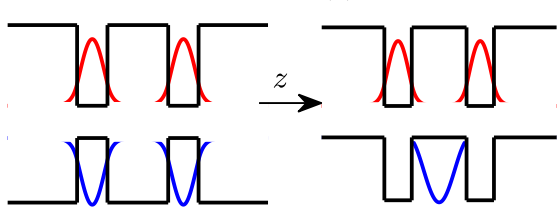

FIG. 1. (a) The nanowire geometry in the double quantum dot (DQD) configuration. The conduction and valence band potential profiles along the nanowire $z$ axis for (b) type-I and (c) type-II DQD geometries.

few particle electron-hole system within the dipole approximation. Coulomb interaction is expected to be a dominating physical effect in the type-II geometries and thus plays a major role in the design of quantum gate devices $[1,7,8,26]$. Other relevant physical effects include strain and piezoelectric effects as well as polarization fields in the wurtzite phase. However, it was shown by Faria Jr. et al. [25] that strain and polarization fields do not have strong influence on the optical properties of type-II InP crystal-phase QDs, and these effects were for this reason neglected in their later work [27]. Similarly, we include Coulomb interaction but exclude all other physical effects to keep the model as simple as possible for our device design purposes.

Enhancement of light-matter interaction is very important for many optical devices [28], such as semiconductor lasers, single-photon sources, detectors, light-emitting diodes, and also for quantum information processing devices. The relevant figure-of-merit in the dipole approximation is the exciton oscillator strength, which is a dimensionless quantity. In this work we show that by engineering a DQD nanowire structure, good spatial confinement of the hole can be restored leading to an exciton oscillator strength several times larger compared to that of the single QD (SQD) geometry. These features make the DQD structure a particularly suitable platform for implementing a quantum gate where the qubits are encoded on neighboring QDs and where the significant oscillator strength is needed in the gating scheme to enable optical control using the stimulated Raman adiabatic passage (STIRAP) process [2,29].

The paper is structured as follows. The details of our model are summarized in Sec. II. We then present results and
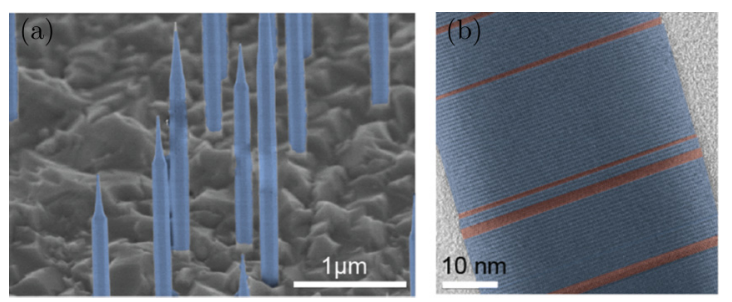

FIG. 2. (a) SEM image of InP nanowires. (b) Crystal-phase QDs formed by zinc blende segments (red) in a wurtzite nanowire [21].

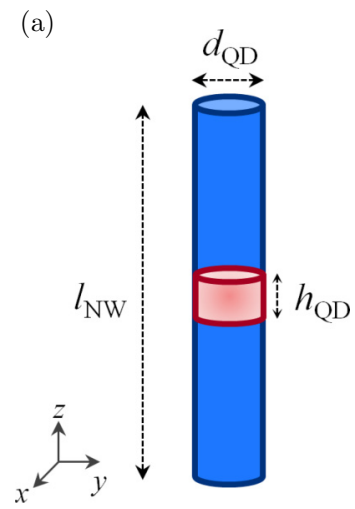

(b)
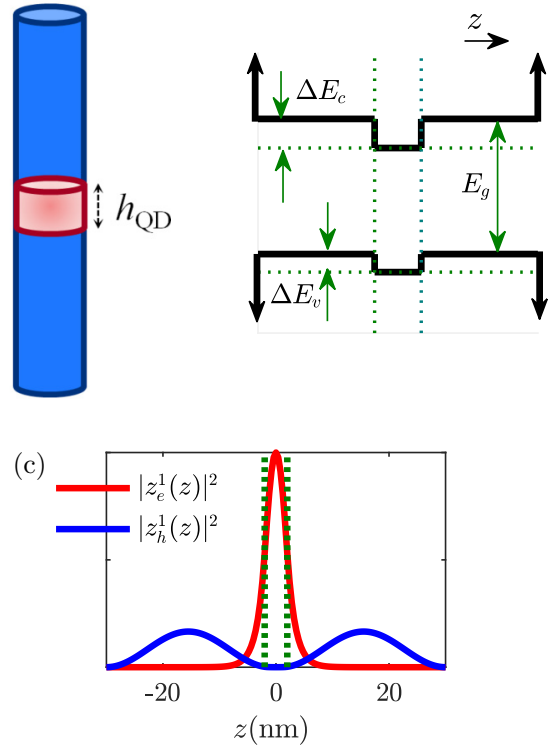

FIG. 3. (a) The type-II SQD nanowire structure. (b) Conduction and valence-band potential energy profiles along the $z$ axis. (c) Ground-state probability densities $\left|Z_{e}^{1}(z)\right|^{2}$ and $\left|Z_{h}^{1}(z)\right|^{2}$ along the $z$ direction for the electron (hole) in the conduction (valence) band The SQD nanowire geometrical parameters in this calculation are: $l_{\mathrm{NW}}=60 \mathrm{~nm}, h_{\mathrm{QD}}=4 \mathrm{~nm}, d_{\mathrm{QD}}=40 \mathrm{~nm}$. The material parameters used in all calculations in this paper are: $\Delta E_{c}=0.129 \mathrm{eV}, \Delta E_{v}=$ $0.0646 \mathrm{eV}, E_{g}=1.474 \mathrm{eV}, m_{e}=0.068 m_{0}$ and $m_{h}=0.64 m_{0}$ [21], and $\epsilon=12.5$ [27].

discuss the limitations of the SQD geometry in Sec. III. The improved performance of the DQD geometry is presented in Sec. IV followed by a conclusion in Sec. V. The details of the derivation of the Coulomb matrix elements and the evaluation of the Coulomb integrals are presented in Appendixes A and B, respectively.

\section{MODELING COULOMB INTERACTION}

We first consider a SQD formed by a thin layer of zinc blende in a wurtzite nanowire as depicted in Fig. 3(a). We then express the exciton Hamiltonian as

$$
\hat{H}=\hat{H}_{h}+\hat{H}_{e}+\hat{H}_{c},
$$

where $\hat{H}_{(e / h)}$ is the single-particle Hamiltonian for the electron/hole and the term $\hat{H}_{c}$ is the Coulomb interaction between the electron and hole. The representation of this operator in terms of creation and annihilation operators is [30]

$$
\hat{H}_{c}=\iint \frac{q_{1} q_{2}}{4 \pi \epsilon} \frac{\hat{\psi}_{e}^{\dagger}(\boldsymbol{r}) \hat{\psi}_{h}^{\dagger}\left(\boldsymbol{r}^{\prime}\right) \hat{\psi}_{h}\left(\boldsymbol{r}^{\prime}\right) \hat{\psi}_{e}(\boldsymbol{r})}{\left|\boldsymbol{r}-\boldsymbol{r}^{\prime}\right|} d \boldsymbol{r} d \boldsymbol{r}^{\prime} .
$$

Here $q_{1}\left(q_{2}\right)$ is the electronic charge of the electron (hole); $\epsilon$ is the permittivity of the material; $\hat{\psi}_{e}^{\dagger}(\boldsymbol{r})\left[\hat{\psi}_{h}^{\dagger}\left(\boldsymbol{r}^{\prime}\right)\right]$ is the field operator which creates an electron (hole) at the position $\boldsymbol{r}$ : $(R, \theta, z)\left[\boldsymbol{r}^{\prime}:\left(R^{\prime}, \theta^{\prime}, z^{\prime}\right)\right]$. The single-particle Hamiltonian in (1) in the effective mass approximation is written in cylindrical 
coordinates as

$$
\hat{H}_{k}=\frac{\hat{p}_{k}^{2}}{2 m_{k}}+V_{T}^{k}(R, \theta)+V_{\mathrm{QW}}^{k}(z),
$$

where $k \in e, h$ is a subscript denoting the electron or hole respectively, $m_{k}$ is the effective mass that we assume to be constant in the whole system; $\hat{p}_{k}$ is the three-dimensional momentum operator; the first term of (3) is the kinetic energy of the electron (hole); $V_{\mathrm{QW}}^{k}(z)$ and $V_{T}^{k}(R, \theta)$ are the decoupled confinement potentials for the electron/hole along the nanowire $z$ axis and in the transverse plane, respectively. We approximate the transverse confinement potential by a hard wall potential:

$$
V_{T}^{k}(R, \theta)= \begin{cases}0 & R<=d_{\mathrm{QD}} / 2, \\ \infty & R>d_{\mathrm{QD}} / 2,\end{cases}
$$

where $d_{\mathrm{QD}}$ is nanowire diameter [cf. Fig. 3(a)]. In the following we consider circularly symmetric InP wurtzite nanowires [21] exclusively. While nanowires with hexagonal cross sections have also been reported [25,27], their individual treatment is beyond the scope of this work.

Using separation of variables in the rotationally symmetric system, the single-particle states are written as $\phi_{k}^{n l m}(\boldsymbol{r})=Z_{k}^{n}(z) R_{k}^{l m}(R, \theta)$. By solving Schrödinger's equation $\hat{H}_{k} \phi_{k}^{n l m}(\boldsymbol{r})=E_{k}^{n l m} \phi_{k}^{n l m}(\boldsymbol{r})$, the transverse part of the singleparticle states is found as $R^{l m}(R, \theta)=A J_{m}\left(\lambda_{m, l} R\right) e^{i m \theta}$ with $A$ being a normalization constant and $\lambda_{m, l}$ the $l$ th-order zero of the Bessel function of the first kind $J_{m}(x)$ of order $m$. The wave function along the $z$ axis $Z_{k}^{n}(z)$ with the potential $V_{\mathrm{QW}}^{k}(z)$ being the single-well band structure shown in Fig. 3(b), is a piecewise function defined differently in each region of the nanowire. The ground-state probabilities of the electron $\left|Z_{e}^{1}(z)\right|^{2}$ and the hole $\left|Z_{h}^{1}(z)\right|^{2}$ are shown in Fig. 3(c). We see that the single-particle hole wave function is mainly localized in the wurtzite material surrounding the QD, with the infinite potential at the nanowire edges shown in Fig. 3(b) being the only confining potential of the single-particle hole state in the SQD nanowire structure.

Our approach for the description of the exciton state is a full configuration-interaction calculation in which the Coulomb Hamiltonian $\hat{H}_{c}$ in (2) is expanded within the Hilbert space spanned by the lowest energy single-particle electron and hole states. Then, the exciton eigenstates $\Psi_{\text {exc }}\left(\boldsymbol{r}_{h}, \boldsymbol{r}_{e}\right)$ and energies $E_{\text {exc }}$ are obtained by direct diagonalization of the Hamiltonian matrix $H$ (see Appendixes A and B). The $\mu$ th excitonic eigenstate is then given by

$$
\Psi_{\mathrm{exc}}^{\mu}\left(\boldsymbol{r}_{e}, \boldsymbol{r}_{h}\right)=\sum_{i, j} C_{i j}^{\mu} \phi_{e}^{i}\left(\boldsymbol{r}_{e}\right) \phi_{h}^{j}\left(\boldsymbol{r}_{h}\right),
$$

where $C_{i j}^{\mu}$ are the elements of the $\mu$ th eigenvector of the Hamiltonian matrix, and $i$ and $j$ are indices summarizing the quantum numbers $(\mathrm{nlm})$ of the single-particle electron and hole states, respectively.

We truncate the Hilbert space of the single-particle electron and hole states in our calculation while ensuring that enough states are included to achieve convergence. For larger structures, the energy spacing of the single-particle states decrease, and an increasing number of higher-order single-particle electron and hole states are needed to correctly represent the

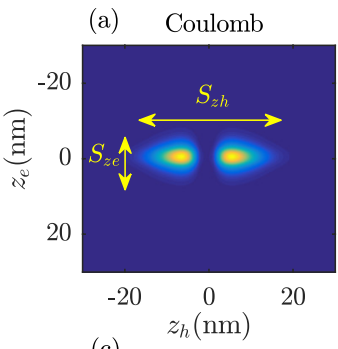

(b) No Coulomb
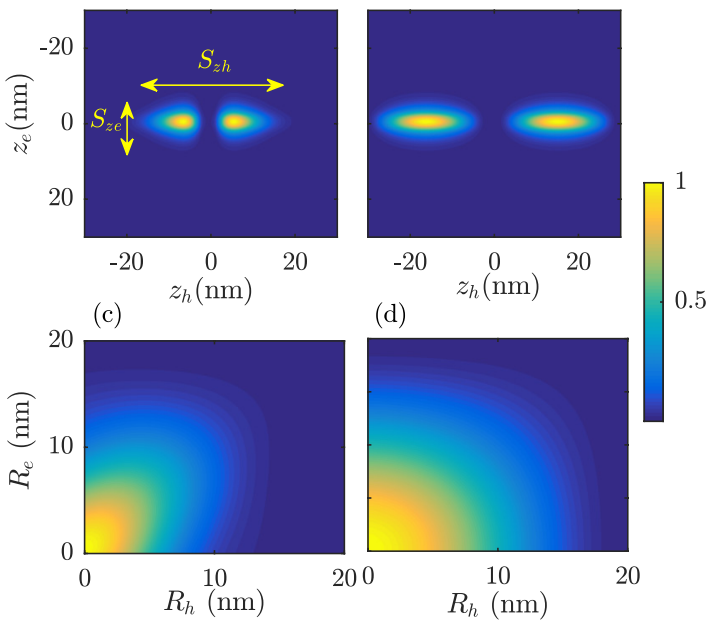

(d)

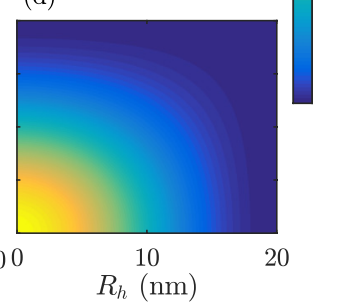

FIG. 4. Ground-state exciton probability density $\left|\Psi_{\text {exc }}\left(\boldsymbol{r}_{h}, \boldsymbol{r}_{e}\right)\right|^{2}$ for $R_{e}=R_{h}=0$ in the (a) presence and (b) absence of Coulomb interaction. Exciton probability for $z_{e}=z_{h}=\theta_{e}=\theta_{h}=0$ in the (c) presence and (d) absence of Coulomb interaction. The geometrical parameters of the SQD nanowire are $d_{\mathrm{QD}}=40 \mathrm{~nm}, h_{\mathrm{QD}}=4 \mathrm{~nm}$, and $l_{\mathrm{NW}}=60 \mathrm{~nm}$.

ground-state exciton wave function and energy as discussed in Appendix B.

The material parameters are listed in the Fig. 3 caption and are taken from [21]. Since the dielectric discontinuity between the two crystal phases is small [27], we use a single constant value of $\epsilon$ in the entire nanowire.

\section{THE SINGLE QUANTUM DOT GEOMETRY}

The ground-state exciton probability density $\left|\Psi_{\text {exc }}\left(\boldsymbol{r}_{h}, \boldsymbol{r}_{e}\right)\right|^{2}$ at $R_{e}=R_{h}=0$ for the SQD geometry is shown in Figs. 4(a) and 4(b) in the presence and absence of Coulomb interaction. We observe that without Coulomb interaction, the hole part of the exciton wave function is spread all over the nanowire. In the presence of Coulomb interaction, the hole is attracted to the electron leading to a hole confined to the surroundings of the QD. Figures 4(c) and 4(d) present the wave function $\left|\Psi_{\text {exc }}\left(\boldsymbol{r}_{h}, \boldsymbol{r}_{e}\right)\right|^{2}$ for $z_{e}=z_{h}=\theta_{e}=\theta_{h}=0$ which corresponds to the radial distribution of the exciton wave function with and without Coulomb interaction, respectively. The figures indicate that Coulomb interaction confines the exciton state in the center of the nanowire, which reduces the influence of unwanted surface potentials due to fabrication imperfections.

We also calculate the exciton oscillator strength, all light polarizations included, given by [13]

$$
O S=\frac{2\left|p_{c v}\right|^{2}}{m_{0} E_{\mathrm{exc}}}\left|\int \Psi_{\mathrm{exc}}(\boldsymbol{r}, \boldsymbol{r}) d \boldsymbol{r}\right|^{2},
$$

where $m_{0}$ is the free electron mass and $p_{c v}$ is the transition matrix element. Figures 5(a) and 5(b) show the normalized oscillator strength $O S_{N}$ vs exciton energy $E_{\mathrm{ex}}$ in the case of including and neglecting the Coulomb interaction, respectively. The normalized exciton oscillator strength is defined as $O S_{N}=O S / O S_{0}$ where $O S_{0}=2\left|p_{c v}\right|^{2} / m_{0} E_{\text {exc }}$ and $E_{\mathrm{ex}}=E_{\mathrm{exc}}-E_{g}$. A closer comparison of the figures 

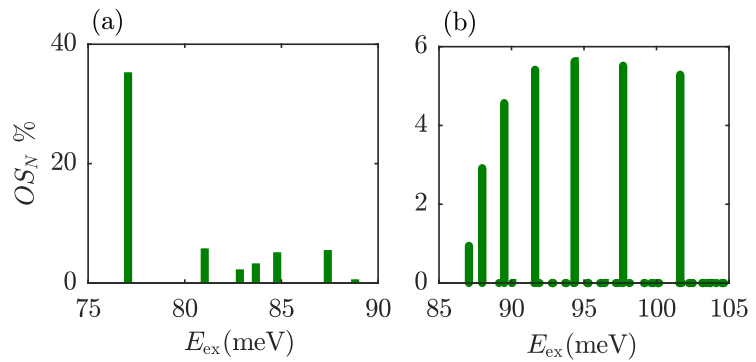

FIG. 5. Normalized oscillator strength in the lowest-energy part (a) when including Coulomb interaction and (b) in the absence of Coulomb interaction. The SQD nanowire geometrical parameters are $d_{\mathrm{QD}}=32 \mathrm{~nm}, h_{\mathrm{QD}}=4 \mathrm{~nm}$, and $l_{\mathrm{NW}}=90 \mathrm{~nm}$.

reveals that the Coulomb interaction is important and should not be neglected: The notable effects are: (1) The attractive Coulomb interaction causes a red shift of the exciton transition energies of around $10 \mathrm{meV}$, which is the exciton binding energy. (2) The Coulomb interaction results in an increase of the ground-state exciton $O S_{N}$ value of more than a factor of 20 compared to the case without Coulomb interaction. (3) While the expansion of the ground-state exciton in the absence of Coulomb interaction simply includes the $\psi_{e}^{100}, \psi_{h}^{100}$ single-particle components, in the presence of Coulomb interaction the dominating contributions in the expansion are the $\psi_{e}^{100}, \psi_{h}^{300}$ and $\psi_{e}^{100}, \psi_{h}^{500}$ components. (4) The energy difference between the ground exciton state and the firstexcited exciton state considerably increases in the presence of Coulomb interaction. It is important because it means there will be less pure dephasing of the ground state due to the virtual phonon decoherence processes [31].

To investigate the influence of the nanowire boundaries on the excitonic properties, we vary the length of the nanowire and study the spatial extent of the electron and hole parts of the wave function. We define the exciton sizes $S_{z e} / S_{z h}$ such that the electron/hole part of the exciton probability is 0.001 of its maximum value along the $z_{e} / z_{h}$ axis when $R_{e}=R_{h}=0$, see Fig. 4(a). Now, Fig. 6(a) reveals that $S_{z h}$ is increasing linearly with the nanowire length $l_{\mathrm{NW}}$ up to $\approx 60 \mathrm{~nm}$ after which the size assumes a constant value independent of the nanowire length. Whereas for smaller nanowires the hole confinement is defined by the nanowire boundary, for $l_{\mathrm{NW}}>60 \mathrm{~nm}$ the confinement mechanism along the nanowire axis is thus dominated by Coulomb attraction to the localized electron. The dependence of the normalized oscillator strength $O S_{N}$ and energy $E_{\text {ex }}$ of the ground-state exciton as a function of the nanowire length is presented in Fig. 6(b). We observe that these parameters also assume length-independent values for nanowire lengths above $\approx 60 \mathrm{~nm}$ similarly to the exciton size in Fig. 6(a). We conclude that when the nanowire length is larger than the exciton hole size along the $z$ axis, the exciton properties become independent of $l_{\mathrm{NW}}$.

The dependence of the ground-state exciton $O S_{N}$ as a function of the nanowire diameter is shown in Fig. 7. When increasing the QD diameter, the electron and hole have more space to move around and the lateral extension of the exciton increases, which means that the overlap integral in (6) increases and, in turn, the oscillator strength. While a large diameter initially appears attractive, on the other hand, by
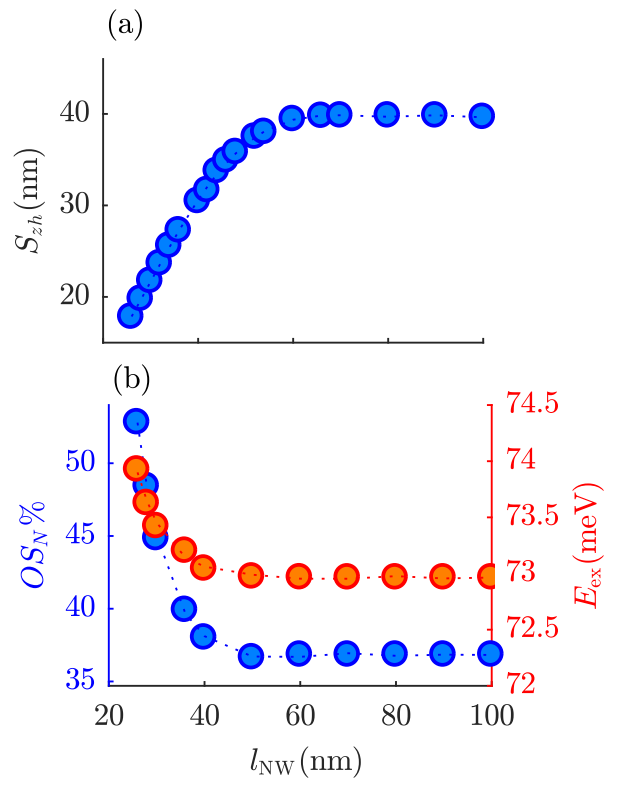

FIG. 6. (a) Hole part of the exciton size $S_{z h}$. (b) Exciton energy $E_{\text {ex }}$ and normalized oscillator strength $O S_{N}$ as a function of the nanowire length $l_{\mathrm{NW}}$ for the ground-state exciton. The SQD nanowire geometrical parameters are $d_{\mathrm{QD}}=40 \mathrm{~nm}$ and $h_{\mathrm{QD}}=4 \mathrm{~nm}$.

increasing the QD diameter the energy separation $\Delta E$ between the ground-state exciton and first-excited state decreases, as is shown in Fig. 7. For a nanowire of diameter above $\approx 60 \mathrm{~nm}$ (with $h_{\mathrm{QD}}=4 \mathrm{~nm}$ ), the difference $\Delta E$ becomes smaller than $1 \mathrm{meV}$, which makes it more challenging to experimentally address the ground-state exciton [31]. This is the first reason that here we are only considering nanowires with diameters up to $60 \mathrm{~nm}$. Furthermore, for sufficiently large diameters, where the exciton size is no longer small compared to the optical wavelength, the dipole approximation breaks down leading to a stabilization of the oscillator strength for increasing diameter [32]. However for the nanowires with $d_{\mathrm{QD}}<60 \mathrm{~nm}$ considered in this paper, the dipole approximation is valid and the oscillator strength increases with diameter as also observed in [32].

The dependence of the exciton sizes $S_{z e}$ and $S_{z h}$ along the electron and hole $z$ axes as function of QD height $h_{\mathrm{QD}}$ is presented in Fig. 8(a). When $h_{\mathrm{QD}}$ decreases, the electron is pushed out of the QD and the exciton electron size $S_{z e}$

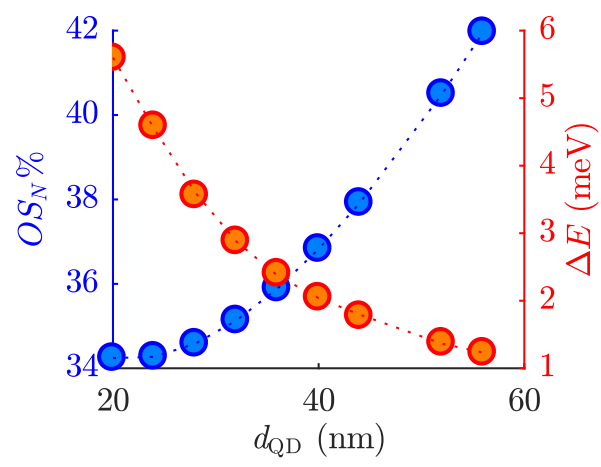

FIG. 7. Ground state exciton normalized oscillator strength $O S_{N}$ and energy separation between the ground-state exciton and firstexcited state $\Delta E$ as function of the QD diameter $d_{\mathrm{QD}}$. The SQD nanowire geometrical parameters are $l_{\mathrm{NW}}=90 \mathrm{~nm}$ and $h_{\mathrm{QD}}=4 \mathrm{~nm}$. 

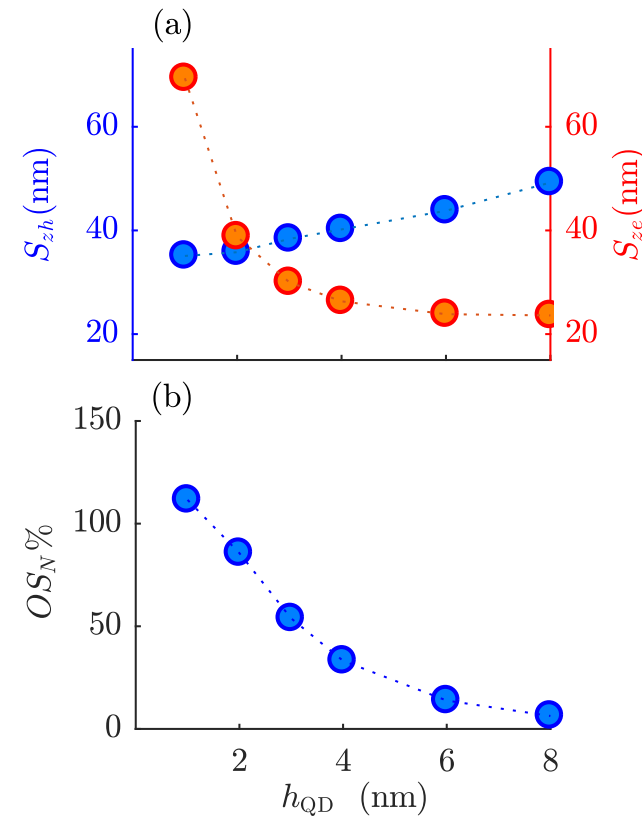

FIG. 8. (a) Hole and electron part of the exciton sizes $S_{z h}$ and $S_{z e}$ and (b) normalized oscillator strength $O S_{N}$ as a function of the QD height $h_{\mathrm{QD}}$ for the ground-state exciton. The SQD nanowire geometrical parameters are $l_{\mathrm{NW}}=90 \mathrm{~nm}$ and $d_{\mathrm{QD}}=20 \mathrm{~nm}$.

increases. In the limit when $h_{\mathrm{QD}} \rightarrow 0$, the electron is strongly delocalized from the QD. This explains why, by decreasing $h_{\mathrm{QD}}$, the exciton $O S_{N}$ increases as observed in Fig. 8(b): By decreasing $h_{\mathrm{QD}}$, the probability of finding the electron and hole on the same site increases, leading to a larger overlap integral (6) and, in turn, an improved oscillator strength.

While a large exciton oscillator strength can clearly be obtained using a large diameter $d_{\mathrm{QD}}$ or a small QD height $h_{\mathrm{QD}}$, we note that in both cases a spatially large exciton is obtained with small energy separation to the first higher-order state. We thus conclude that the SQD geometry is not ideal for quantum gating applications. In the next section, we show that by engineering a DQD nanowire structure, it is possible to improve the exciton oscillator strength while maintaining a spatially well-confined profile.

Finally, while a symmetric nanowire geometry in which the QD is placed in the center of the nanowire was considered for all the SQD calculations presented in this section, for realistic SQD nanowires the QD may not be positioned exactly in the center. However, we have demonstrated that the exciton is confined by the Coulomb interaction for nanowire lengths above $\approx 60 \mathrm{~nm}$. Thus, for a QD separated by more than $30 \mathrm{~nm}$ from the closest nanowire termination, the asymmetry is not expected to play any role.

\section{THE DOUBLE QUANTUM DOT STRUCTURE}

While excitons in isolated QDs are generally limited to one or two qubit operations, the need for scalable qubit arrays has led to proposals of few-QD nanostructures like DQDs for physical realization of universal quantum logic gates [33,34]. Additionally, pairs of vertically aligned type-I QDs for optically driven solid state quantum gates [35-37]

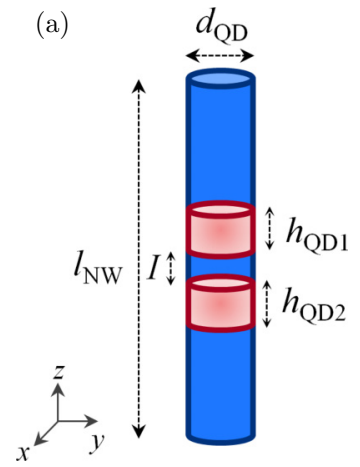

(b)

FIG. 9. (a) The type-II DQD nanowire structure. (b) Probability functions along the nanowire $z$ axis for the ground-state electron in the conduction-band $\left|Z_{e}^{1}(z)\right|^{2}$ and for the lowest-energy bound-state hole in the valence-band $\left|Z_{h}^{b}(z)\right|^{2}$. The DQD nanowire geometrical parameters are $l_{\mathrm{NW}}=60 \mathrm{~nm}, h_{\mathrm{QD} 1}=h_{\mathrm{QD} 2}=4 \mathrm{~nm}, I=8 \mathrm{~nm}$, and $d_{\mathrm{QD}}=20 \mathrm{~nm}$.

have been suggested. We will now show that type-II DQDs in nanowires are also promising for implementing optically controlled quantum gates.

In the DQD nanowire structure shown in Fig. 9(a), the single-particle electron states are confined to the QDs as for the SQD configuration. Additionally, most single-particle hole states are predominantly localized in the outer nanowire regions surrounding the QDs. However, there are a few single-particle hole states which mainly are localized in the barrier region between two QDs, which we in the following refer to as bound-state holes. The wave functions for the singleparticle ground-state electron and lowest-energy bound-state hole are shown in Fig. 9(b), and we observe that the DQD

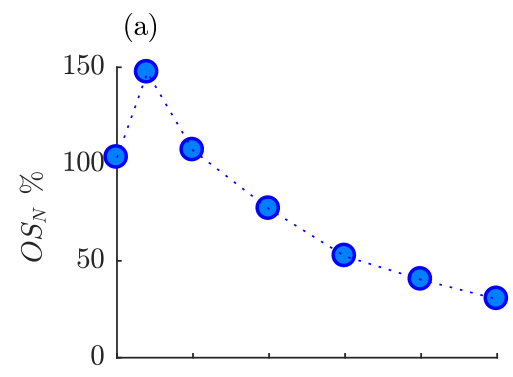

(b)

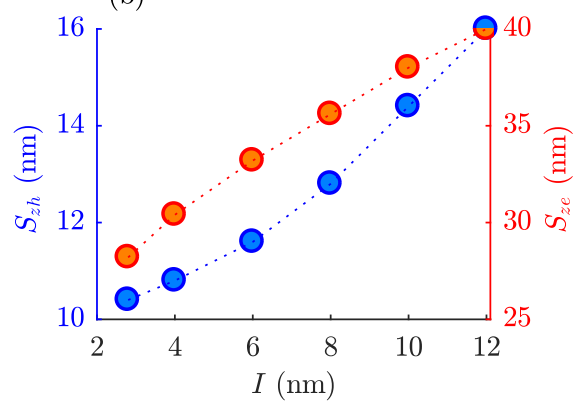

FIG. 10. (a) Normalized oscillator strength $O S_{N}$ for the lowestenergy bound-state exciton. (b) Size $S_{z h}$ along the $z_{h}$ axis and $S_{z e}$ along the $z_{e}$ axis as a function of inner dot separation $I$. The DQD nanowire geometrical parameters are $l_{\mathrm{NW}}=90 \mathrm{~nm}, d_{\mathrm{QD}}=52 \mathrm{~nm}$, and $h_{\mathrm{QD} 1}=h_{\mathrm{QD} 2}=4 \mathrm{~nm}$. 


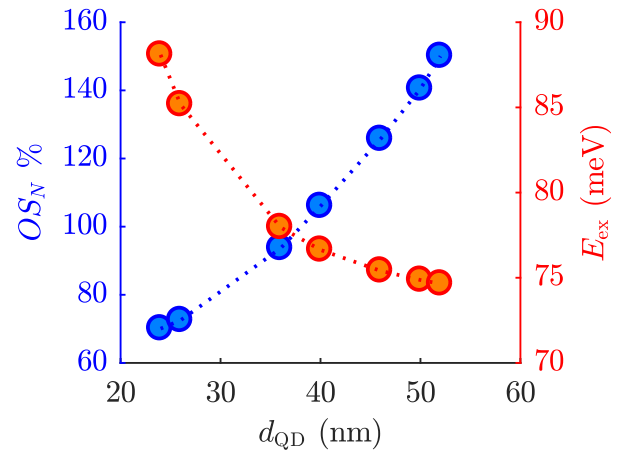

FIG. 11. Normalized oscillator strength $O S_{N}$ and energy $E_{\mathrm{ex}}$ as a function of the nanowire diameter $d_{\mathrm{QD}}$ for the lowest-energy bound-state exciton. The DQD nanowire geometrical parameters are $l_{\mathrm{NW}}=90 \mathrm{~nm}, h_{\mathrm{QD} 1}=h_{\mathrm{QD} 2}=4 \mathrm{~nm}$, and $I=2.8 \mathrm{~nm}$.

configuration allows for significantly improved electron-hole overlap as compared to the SQD configuration due to the strong localization of both the electron and the hole wave functions to the inner DQD region.

We first study the influence of the inner dot separation distance $I$ on the exciton properties. The normalized oscillator strength of the lowest-energy bound-state exciton $O S_{N}$ as a function of $I$ is presented in Fig. 10(a). We observe an optimum value of $I$ where the best trade-off between bound-hole leakage through the QD barriers and electron state penetration into the central barrier is obtained. Here the $O S_{N}$ value of the exciton transition is maximized and is more than 4 times larger than that of the ground-state exciton in a SQD nanowire for the same values of $h_{\mathrm{QD}}$ and $d_{\mathrm{QD}}$. Figure 10(b) shows the exciton electron and hole sizes along the $z_{e}$ and $z_{h}$ axes. We observe that the exciton is significantly more localized along the $z_{h}$ axis compared to the SQD nanowire [cf. Fig. 8(a)]. This feature of the DQD nanowire is a main asset making it highly suitable as a platform for optically controlled quantum gates.

The dependence of the bound exciton normalized oscillator strength $O S_{N}$ and energy $E_{\text {ex }}$ on the QD diameter $d_{\mathrm{QD}}$ is depicted in Fig. 11. As for the SQD geometry, we observe that $O S_{N}$ increases with diameter within the dipole approximation. By choosing a DQD nanowire of $\approx 50 \mathrm{~nm}$, we can achieve a large oscillator strength, which is a key parameter in quantum

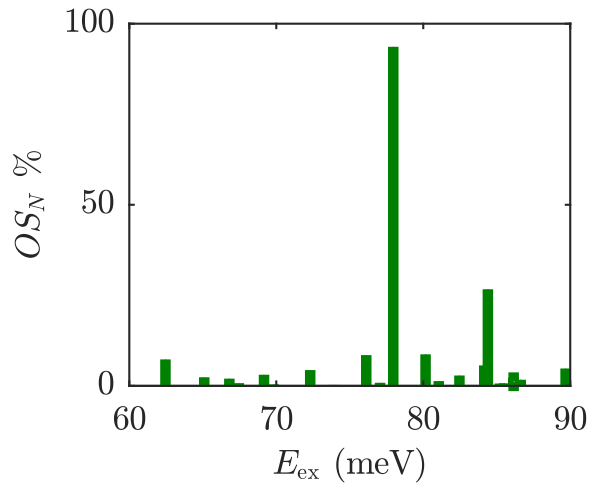

FIG. 12. The exciton normalized oscillator strength in the lowest-energy part. The DQD nanowire geometrical parameters are $l_{\mathrm{NW}}=90 \mathrm{~nm}, h_{\mathrm{QD} 1}=h_{\mathrm{QD} 2}=4 \mathrm{~nm}, I=2.8 \mathrm{~nm}$, and $d_{\mathrm{QD}}=36 \mathrm{~nm}$.

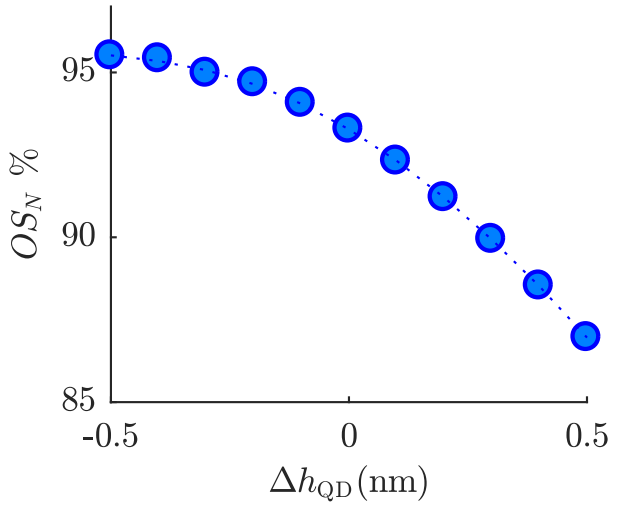

FIG. 13. The bound-state exciton normalized oscillator strength $O S_{N}$ as a function of the difference between the height of the two quantum dots in the DQD structure $\Delta h_{\mathrm{QD}}=h_{\mathrm{QD} 2}-h_{\mathrm{QD} 1}$. The DQD nanowire geometrical parameters are $l_{\mathrm{NW}}=90 \mathrm{~nm}, I=2.8 \mathrm{~nm}$, $d_{\mathrm{QD}}=36 \mathrm{~nm}$, and $h_{\mathrm{QD} 1}=4 \mathrm{~nm}$.

gates. Again, the diameter should be chosen as a trade-off between large oscillator strength and sufficient energy level difference between the exciton and the first higher order boundstate exciton as discussed in Sec. III.

The exciton normalized oscillator strength in the lowest energy part for the DQD configuration is presented in Fig. 12. We observe a dominating peak at $\approx 78 \mathrm{meV}$, which corresponds to the lowest-energy bound-state exciton. The oscillator strength of the bound exciton is thus much stronger than other excitonic transitions in the lowest-energy part of the excitonic spectrum for the DQD structure. This feature makes selective excitation of the bound-state exciton feasible in experiments. Also since the real-space overlap between the interesting bound-state exciton and close-in-energy unbound-state excitons is small, phonon decay processes can be neglected [5].

For realistic structures, asymmetry of the DQD geometry can occur due to a nonideal fabrication process. We study in Fig. 13 the influence of the QDs height difference $\Delta h_{\mathrm{QD}}=$ $h_{\mathrm{QD} 2}-h_{\mathrm{QD} 1}$ on the oscillator strength of the lowest energy bound-state exciton for a fixed QD1 height of $h_{\mathrm{QD} 1}=4 \mathrm{~nm}$. We observe that upon increasing the height of QD2 by $0.5 \mathrm{~nm}$ the oscillator strength decreases by $6 \%$, while a height decrease by $0.5 \mathrm{~nm}$ leads to an increase of the oscillator strength by $2 \%$.

\section{CONCLUSION}

Using an efficient method based on a configurationinteraction description, we have analyzed the main properties of excitons in type-II single and double quantum dots in nanowires. Energy spectra, oscillator strengths, and electron and hole exciton sizes have been calculated as a function of all relevant geometrical parameters.

The Coulomb interaction is sufficient to bind the hole part of the exciton to the QD in a single-quantum dot geometry, such that the exciton properties become insensitive to the length for nanowire lengths larger than the exciton size. However, in the single-quantum dot geometry the oscillator strength of the ground-state exciton is significantly reduced compared to a type-I system. While the oscillator strength of the exciton transition in a single-quantum dot in the limit of infinitely small 
QD height increases, the exciton is not spatially confined to the QD and thus not suitable for quantum gate applications.

We have then proposed a double-quantum dot structure for which the exciton oscillator strength can be increased to more than four times its value compared to that of a single-quantum dot nanowire while the exciton remains well-confined to the double-quantum dot region. This structure featuring a combination of separated electron and hole localization and a large exciton oscillator strength represents a promising platform for implementing two-qubit quantum gates.

\section{ACKNOWLEDGMENTS}

This project has received funding from the Danish National Research Council for Technology and Production, 4005-00370, 703193 and the European Union's Horizon 2020 research and innovation programme under the Marie Skłodowska-Curie Grant Agreement No. 703193.

\section{APPENDIX A: COULOMB MATRIX ELEMENTS}

To calculate the matrix elements of $H_{c}$ in (2), the action of the Coulomb Hamiltonian on a state vector should be evaluated. For this purpose, we write a state vector using field operators as

$$
|\phi\rangle=\int d \boldsymbol{r} \phi(\boldsymbol{r}) \hat{\psi}^{\dagger}(\boldsymbol{r})|0\rangle=\int d \boldsymbol{r} \phi(\boldsymbol{r})|\boldsymbol{r}\rangle .
$$

By applying the annihilation field operator on the state vector and by using the commutation relation for the field operators $\left[\hat{\psi}(\boldsymbol{r}), \hat{\psi}^{\dagger}\left(\boldsymbol{r}^{\prime}\right)\right]=\delta\left(\boldsymbol{r}-\boldsymbol{r}^{\prime}\right)$, we obtain

$$
\hat{\psi}(\boldsymbol{r})|\phi\rangle=\int d \boldsymbol{r}^{\prime} \phi\left(\boldsymbol{r}^{\prime}\right) \hat{\psi}(\boldsymbol{r}) \hat{\psi}^{\dagger}\left(\boldsymbol{r}^{\prime}\right)|0\rangle=\phi(\boldsymbol{r})|0\rangle .
$$

The matrix elements of the Hamiltonian operator $\hat{H}_{c}$ in (2) can then be evaluated as

$$
\begin{aligned}
\left\langle i^{\prime} j^{\prime}\left|\hat{H}_{c}\right| i j\right\rangle= & \frac{q_{1} q_{2}}{4 \pi \epsilon} \iint\left\langle i^{\prime} j^{\prime}\left|\frac{\hat{\psi}_{e}^{\dagger}(\boldsymbol{r}) \hat{\psi}_{h}^{\dagger}\left(\boldsymbol{r}^{\prime}\right) \hat{\psi}_{h}\left(\boldsymbol{r}^{\prime}\right) \hat{\psi}_{e}(\boldsymbol{r})}{\left|\boldsymbol{r}-\boldsymbol{r}^{\prime}\right|}\right| i j\right\rangle \\
& \times d \boldsymbol{r} d \boldsymbol{r}^{\prime} .
\end{aligned}
$$

In the above expression, we first evaluate the annihilation operators acting on the two-particle state vector

$$
\begin{aligned}
\hat{\psi}_{h}\left(\boldsymbol{r}_{h}\right) \hat{\psi}_{e}\left(\boldsymbol{r}_{e}\right)|i j\rangle= & \int d \boldsymbol{r}_{e} \int d \boldsymbol{r}_{h} \phi_{e}^{i}\left(\boldsymbol{r}_{e}\right) \phi_{h}^{j}\left(\boldsymbol{r}_{h}\right) \hat{\psi}_{h}\left(\boldsymbol{r}^{\prime}\right) \hat{\psi}_{e}(\boldsymbol{r}) \\
& \times \hat{\psi}_{e}^{\dagger}\left(\boldsymbol{r}_{e}\right) \hat{\psi}_{h}^{\dagger}\left(\boldsymbol{r}_{h}\right)|0\rangle .
\end{aligned}
$$

Since we have

$$
\hat{\psi}_{h}\left(\boldsymbol{r}^{\prime}\right) \hat{\psi}_{e}(\boldsymbol{r}) \hat{\psi}_{e}^{\dagger}\left(\boldsymbol{r}_{e}\right) \hat{\psi}_{h}^{\dagger}\left(\boldsymbol{r}_{h}\right)|0\rangle=\delta\left(\boldsymbol{r}-\boldsymbol{r}_{e}\right) \delta\left(\boldsymbol{r}^{\prime}-\boldsymbol{r}_{h}\right)|0\rangle,
$$

Eq. (A4) can be simplified as

$$
\hat{\psi}_{h}\left(\boldsymbol{r}^{\prime}\right) \hat{\psi}_{e}(\boldsymbol{r})|i j\rangle=\phi_{e}^{i}(\boldsymbol{r}) \phi_{h}^{j}\left(\boldsymbol{r}^{\prime}\right)|0\rangle .
$$

Similarly for the bra parts we obtain

$$
\begin{aligned}
\left\langle i^{\prime} j^{\prime}\right| \hat{\psi}_{e}^{\dagger}(\boldsymbol{r}) \hat{\psi}_{h}^{\dagger}\left(\boldsymbol{r}^{\prime}\right) & =\left\{\hat{\psi}_{h}\left(\boldsymbol{r}^{\prime}\right) \hat{\psi}_{e}(\boldsymbol{r})\left|i^{\prime} j^{\prime}\right\rangle\right\}^{\dagger} \\
& =\langle 0| \phi_{e}^{i^{\prime} *}(\boldsymbol{r}) \phi_{h}^{j^{\prime} *}\left(\boldsymbol{r}^{\prime}\right) .
\end{aligned}
$$

By replacing (A6) and (A7) and $q_{1}=-e$ and $q_{2}=e$ in (A3), the matrix elements become

$$
\left\langle i^{\prime} j^{\prime}\left|\hat{H}_{c}\right| i j\right\rangle=\frac{-e^{2}}{4 \pi \epsilon} \iint \frac{\phi_{e}^{i^{\prime} *}(\boldsymbol{r}) \phi_{h}^{j^{\prime *} *}\left(\boldsymbol{r}^{\prime}\right) \phi_{e}^{i}(\boldsymbol{r}) \phi_{h}^{j}\left(\boldsymbol{r}^{\prime}\right)}{\left|\boldsymbol{r}-\boldsymbol{r}^{\prime}\right|} d \boldsymbol{r} d \boldsymbol{r}^{\prime}
$$

\section{APPENDIX B: CALCULATION OF THE COULOMB INTEGRAL}

In this Appendix we discuss the calculation of the (A8) integral. Its evaluation is not straightforward due to the divergence at $\boldsymbol{r}=\boldsymbol{r}^{\prime}$. However, we can write $\frac{1}{\left|\boldsymbol{r}-\boldsymbol{r}^{\prime}\right|}$ as a generating function [38] of circular cylindrical harmonics as

$$
\frac{1}{\left|\boldsymbol{r}-\boldsymbol{r}^{\prime}\right|}=\sum_{s=-\infty}^{\infty} e^{i s\left(\theta-\theta^{\prime}\right)} \int_{0}^{\infty} J_{s}(k R) J_{s}\left(k R^{\prime}\right) e^{-k\left|z-z^{\prime}\right|} d k .
$$

By inserting (B1) and the single-particle state vectors $\phi_{k}^{n l m}$ as discussed in the main text into (A8), and subsequently integrating over $\theta$ and summing over $s$, the Coulomb integral (A8) becomes

$$
\begin{aligned}
\left\langle i^{\prime} j^{\prime}\left|\hat{H}_{c}\right| i j\right\rangle= & \frac{-e^{2}}{4 \pi \epsilon} \delta_{m_{i}-m_{i^{\prime}}, m_{j^{\prime}}-m_{j}} \\
& \times \iiint_{z, z^{\prime}, k} d k d z d z^{\prime} e^{-k\left|z-z^{\prime}\right|} Z_{e}^{n_{i^{\prime}} *}(z) \\
& \times Z_{h}^{n_{j^{\prime}} *}\left(z^{\prime}\right) Z_{e}^{n_{i}}(z) Z_{h}^{n_{j}}\left(z^{\prime}\right) \\
& \times \int_{R} R d R J_{m_{i}-m_{i^{\prime}}}(k R) R_{e}^{l_{i^{\prime}}, m_{i^{\prime}} *}(R) R_{e}^{l_{i}, m_{i}}(R) \\
& \times \int_{R^{\prime}} R^{\prime} d R^{\prime} J_{m_{j^{\prime}}-m_{j}}\left(k R^{\prime}\right) \\
& \times R_{h}^{l_{j^{\prime}}, m_{j^{\prime}} *}\left(R^{\prime}\right) R_{h}^{l_{j}, m_{j}}\left(R^{\prime}\right) .
\end{aligned}
$$

Now the integral is separated and can be evaluated numerically. The integrals over $R, R^{\prime}, z$, and $z^{\prime}$ are straightforward. The remaining one-dimensional integral over $k$ is performed by first truncating the integral using a cut-off $k_{\text {cut }}$ and subsequently discretizing it with a discretization step $\Delta k$. When introducing the truncation and the discretization, careful convergence studies are required. Typical convergence studies for the cut-off $k_{\text {cut }}$ and the discretization step $\Delta k$ are presented in Fig. 14. Here we study the ground-state exciton energy (the lowest eigenvalue of the Hamiltonian matrix) and its oscillator strength, which is calculated using the Hamiltonian matrix eigenvector corresponding to the lowest eigenvalue and using the single-particle states according to (5) and (6). Additionally, Fig. 15 shows a typical convergence study of the exciton oscillator strength and energy as function of the number $N_{T}$ which is equal to $N_{T e}\left(N_{T h}\right)$ of in-plane transverse electron (hole) single-particle states $\left[R_{k}^{l m}(R, \theta)\right]$ for two diameters $d_{\mathrm{QD}}=32 \mathrm{~nm}$ and $d_{\mathrm{QD}}=40 \mathrm{~nm}$.

The figure reveals that the ground-state exciton energy $E_{\mathrm{ex}}$ converges much faster than the $O S_{N}$ : The variation of the exciton $O S_{N}$ is around $200 \%$ in the total interval considered, whereas the variation for the exciton energy is less than $2 \%$. As the QD diameter increases, the energy spacing between 

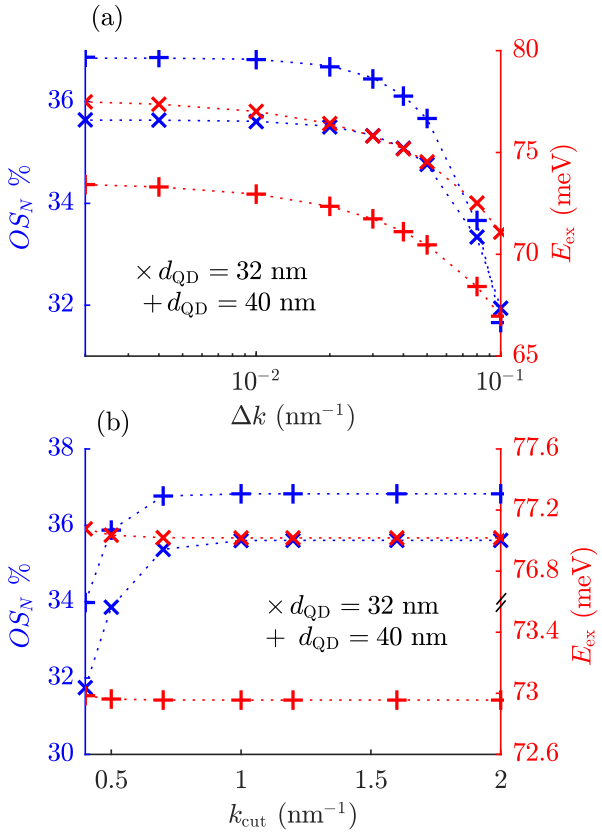

FIG. 14. Convergence study of the ground-state exciton $O S_{N}$ and $E_{\text {ex }}$ as function of (a) $\Delta k$ with $k_{\text {cut }}=1 \mathrm{~nm}^{-1}$ and as function of (b) $k_{\text {cut }}$ with $\Delta k=0.01 \mathrm{~nm}^{-1}$. The SQD nanowire geometrical parameters are $l_{\mathrm{NW}}=70 \mathrm{~nm}$ and $h_{\mathrm{QD}}=4 \mathrm{~nm}$. We consider two cases with $d_{\mathrm{QD}}=$ $32 \mathrm{~nm}$ (crosses) and $d_{\mathrm{QD}}=40 \mathrm{~nm}$ (pluses).

the single-particle electron and hole states in the transverse in-plane direction become comparable or smaller than the ground-state exciton binding energy and we need to include

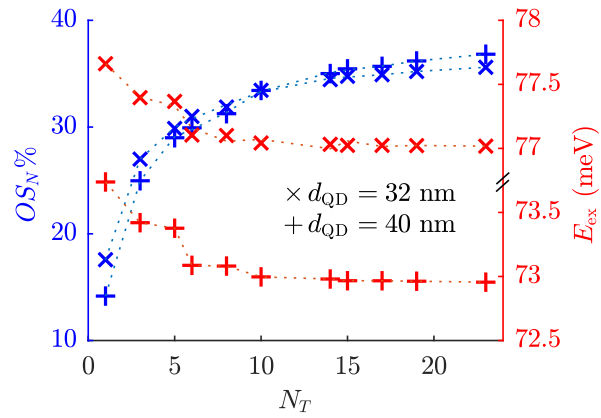

FIG. 15. Convergence study of the ground-state exciton $O S_{N}$ and $E_{\mathrm{ex}}$ on the number $N_{T}=N_{T e}=N_{T h}$ of in-plane transverse single-particle electron (hole) states for two diameters $d_{\mathrm{QD}}=32 \mathrm{~nm}$ (crosses) and $d_{\mathrm{QD}}=40 \mathrm{~nm}$ (pluses). The SQD nanowire structure parameters are $l_{\mathrm{NW}}=70 \mathrm{~nm}$ and $h_{\mathrm{QD}}=4 \mathrm{~nm}$. In this calculation we considered $N_{z e}=2$ and $N_{z h}=20$ for the single-particle electron and hole states along $z$ axis, respectively, and we have $\Delta k=0.01 \mathrm{~nm}^{-1}$ and $k_{\mathrm{cut}}=1 \mathrm{~nm}^{-1}$

more in-plane single-particle states in the calculation: Closer inspection of Fig. 15 reveals convergence is slower for the $d_{\mathrm{QD}}=40 \mathrm{~nm}$ diameter than for the $d_{\mathrm{QD}}=32 \mathrm{~nm}$ case. The same argument also applies for other geometrical parameters like the nanowire length. As the nanowire length increases, the energy spacing of the single-particle hole states along the $z$ axis is reduced and additional higher single-particle hole states in $z$ direction contribute to the ground-state exciton wave function and energy. Similarly, for QDs of increasing height, the energy separation of the single-particle electron states is reduced and more states are needed to obtain convergence.
[1] E. Biolatti, R. C. Iotti, P. Zanardi, and F. Rossi, Phys. Rev. Lett. 85, 5647 (2000).

[2] F. Troiani, E. Molinari, and U. Hohenester, Phys. Rev. Lett. 90, 206802 (2003).

[3] H. Kamada and H. Gotoh, Semicond. Sci. Technol. 19, S392 (2004).

[4] N. H. Bonadeo, J. Erland, D. Gammon, D. Park, D. S. Katzer, and D. G. Steel, Science 282, 1473 (1998).

[5] U. Bockelmann and G. Bastard, Phys. Rev. B 42, 8947 (1990).

[6] D. S. Citrin, Phys. Rev. B 47, 3832 (1993).

[7] X. Li, Y. Wu, D. Steel, D. Gammon, T. H. Stievater, D. S. Katzer, D. Park, C. Piermarocchi, and L. J. Sham, Science 301, 5634 (2003).

[8] B. W. Lovett, J. H. Reina, A. Nazir, and G. A. D. Briggs, Phys. Rev. B 68, 205319 (2003).

[9] M. Cai, Y. Liu, W. Liu, and T. Yu, Physica B (Amsterdam) 176, 327 (1992)

[10] K. Pemasiri, M. Montazeri, R. Gass, L. M. Smith, H. E. Jackson, J. Yarrison-Rice, S. Paiman, Q. Gao, H. Hoe Tan, C. Jagadish, X. Zhang, and J. Zou, Nano Lett. 9, 648 (2009).

[11] G. Ortner, M. Bayer, Y. Lyanda-Geller, T. L. Reinecke, A. Kress, J. P. Reithmaier, and A. Forchel, Phys. Rev. Lett. 94, 157401 (2005).

[12] G. E. W. Bauer and T. Ando, Phys. Rev. B 38, 6015 (1988).

[13] G. W. Bryant, Phys. Rev. B 37, 8763 (1988).
[14] G. Bastard, E. E. Mendez, L. L. Chang, and L. Esaki, Phys. Rev. B 26, 1974 (1982).

[15] A. Zrenner, E. Beham, S. Stufler, F. Findeis, M. Bichler, and G. Abstreiter, Nature (London) 418, 612 (2002).

[16] A. Barenco, D. Deutsch, A. Ekert, and R. Jozsa, Phys. Rev. Lett. 74, 4083 (1995).

[17] N. Akopian, G. Patriarche, L. Liu, J. C. Harmand, and V. Zwiller, Nano Lett. 10, 1198 (2010).

[18] P. Corfdir and P. Lefebvre, J. Appl. Phys. 112, 053512 (2012).

[19] G. Jacopin, L. Rigutti, L. Largeau, F. Fortuna, F. Furtmayr, F. H. Julien, M. Eickhoff, and M. Tchernycheva, J. Appl. Phys. 110, 064313 (2011).

[20] L. Zhang, J. W. Luo, A. Zunger, N. Akopian, V. Zwiller, and J. C. Harmand, Nano Lett. 10, 4055 (2010).

[21] M. B. Bavinck, K. D. Jns, M. Zieliski, G. Patriarche, J. C. Harmand, N. Akopian, and V. Zwiller, Nano Lett. 16, 1081 (2016).

[22] P. Zanardi and F. Rossi, Phys. Rev. Lett. 81, 4752 (1998).

[23] F. Troiani, U. Hohenester, and E. Molinari, Phys. Rev. B 62, R2263 (2000).

[24] O. Marquardt, M. Ramsteiner, P. Corfdir, L. Geelhaar, and O. Brandt, Phys. Rev. B 95, 245309 (2017).

[25] P. E. Faria, Jr. and G. M. Sipahi, arXiv:1202.2804.

[26] S. D. Rinaldis, I. D’Amico, and F. Rossi, Appl. Phys. Lett. 81, 4236 (2002). 
[27] P. E. Faria, Jr. and G. M. Sipahi, J. Appl. Phys. 112, 103716 (2012).

[28] P. Tighineanu, R. Daveau, E. H. Lee, J. D. Song, S. Stobbe, and P. Lodahl, Phys. Rev. B 88, 155320 (2013).

[29] J. R. Kuklinski, U. Gaubatz, F. T. Hioe, and K. Bergmann, Phys. Rev. A 40, 6741 (1989).

[30] F. Troiani, U. Hohenester, and E. Molinari, Phys. Rev. B 65, 161301(R) (2002).

[31] A. Reigue, J. Iles-Smith, F. Lux, L. Monniello, M. Bernard, F. Margaillan, A. Lemaitre, A. Martinez, D. P. S. McCutcheon, J. Mørk, R. Hostein, and V. Voliotis, Phys. Rev. Lett. 118, 233602 (2017).

[32] S. Stobbe, P. T. Kristensen, J. E. Mortensen, J. M. Hvam, J. Mørk, and P. Lodahl, Phys. Rev. B 86, 085304 (2012).
[33] H. J. Krenner, M. Sabathil, E. C. Clark, A. Kress, D. Schuh, M. Bichler, G. Abstreiter, and J. J. Finley, Phys. Rev. Lett. 94, 057402 (2005).

[34] E. A. Stinaff, M. Scheibner, A. S. Bracker, I. V. Ponomarev, V. L. Korenev, M. E. Ware, M. F. Doty, T. L. Reinecke, and D. Gammon, Science 311, 636 (2006).

[35] L. Robledo, J. Elzerman, G. Jundt, M. Atatre, A. Hgele1, S. Flt, and A. Imamoglu, Science 320, 772 (2008).

[36] O. Gywat, G. Burkard, and D. Loss, Phys. Rev. B 65, 205329 (2002).

[37] M. Bayer, P. Hawrylak, K. Hinzer, S. Fafard, M. Korkusinski, Z. R. Wasilewski, O. Stern, and A. Forchel, Science 291, 451 (2001).

[38] L. Medina and E. Ley Koo, Rev. Mex. Fis. E 54(2), 153 (2008). 